MRS HEATHER DAWSON (Orcid ID : 0000-0001-7527-336X)

MR MICHA DAVID EICHMANN (Orcid ID : 0000-0001-6283-659X)

Article type : Original Article

Tumour Budding/T-cell infiltrates in Colorectal Cancer: Proposal of a Novel Combined Score

Running title: Tumour buds and T-cells in colorectal cancer

Heather Dawson $^{1 *}$, Lucine Christe ${ }^{1 *}$, Micha Eichmann${ }^{1}$, Stefan Reinhard ${ }^{1}$, Inti Zlobec ${ }^{1}$, Annika Blank ${ }^{1}$, Alessandro Lugli ${ }^{1}$

* Equally contributing first authors

${ }^{1}$ Institute of Pathology, University of Bern, Bern, Switzerland

\title{
Disclosure/conflict of interest
}

All authors declare no conflicts of interest, including any financial, personal or other relationships with other people or organisations within that could have inappropriately influenced (biased) their work.

This article has been accepted for publication and undergone full peer review but has not been through the copyediting, typesetting, pagination and proofreading process, which may lead to differences between this version and the Version of Record. Please cite this article as doi: 10.1111/HIS.14006

This article is protected by copyright. All rights reserved 


\section{Word count: 2666}

\section{Corresponding Author}

Dr. Heather Dawson, MD

Institute of Pathology

University of Bern

Murtenstr. 31, P.O. Box 62,

CH-3008 Bern, Switzerland

Phone +41316323211

Fax +41316320969

E-Mail: heather.dawson@pathology.unibe.ch

\section{Abstract (word count 248, max. 250)}

Aims: The TNM classification system is used for prognostication purposes and to guide patient management. However, in colorectal cancer (CRC), additional markers are needed to stratify prognostic subgroups. From large bodies of research, two promising markers have emerged: Tumour budding and T-cell host response (CD3, CD8 and CD45RO infiltrates). However, attempts to combine these two parameters have been sparse. The aim of this study was to perform an assessment of potential protagonists that could be used in a combined score (Budding/T-cell Score, BTS).

Methods and Results: This descriptive, retrospective study was performed on a multi-punch tissue microarray containing material from 345 patients with Stage I-IV CRC. Areas from tumour centre, 
front and microenvironment were stained for Pancytokeratin/CD3, Pancytokeratin/CD8 and Pancytokeratin/CD45RO. Tumour buds were scored manually and T-cell infiltrates digitally using open-source software (QuPath). Tumour buds, T-cell counts and combined BTS were associated with clinico-pathological features and overall survival (OS).

A higher combined BTS score (Buds/CD8, tumour centre) performed better than budding or CD8/CD3 alone in predicting nodal metastases ( $\mathrm{p}<0.0001$, OR 1.466, 95\%CI: 1.115-1.928). Only higher BTS (Buds/CD3) was significantly associated with poorer OS on multivariate analysis ( $\mathrm{p}=$ 0.012, HR 1.218, 95\%CI: 1.044-1.419).

Conclusions: Although CD8+/CD3+ T-cells are predictive of tumour biology in CRC, we found a combined BTS to be stronger in predicting survival and certain features with high clinical relevance, such as nodal metastases, in comparison to budding or T-cells alone. Further studies combining T-cell infiltrates and tumour budding are necessary to optimize risk assessment of CRC.

Keywords: Tumour budding, colorectal cancer, host response, Immunoscore 


\section{Introduction}

The TNM staging system remains the main classification system used to determine prognosis and therapy in cancer patients. However, as the TNM classification in colorectal cancer (CRC) is quite heterogeneous in different stages ${ }^{1,2}$, additional parameters have been proposed to complement this system. Here, the concepts of tumour budding ${ }^{3,4}$ and Immunoscore 5, 6 have emerged as particularly promising candidates.

Many studies have investigated tumour budding, defined as single tumour cells and small clusters of four or less tumour cells as a robust predictor of lymph node and distant metastasis, relapse and poorer outcome ${ }^{7-9}$. These strong associations can be exploited in specific clinical scenarios ${ }^{10}$. A recent proposal of standardized assessment of tumour budding (according to the International Tumor Budding Consensus Conference 2016, ITBCC) ${ }^{10}$ has led to its integration in the CRC checklist of the College of American Pathologists ${ }^{11}$, and widespread reporting of this feature can be expected in the future. From a biological perspective, tumour budding may represent the morphological correlate of epithelial-mesenchymal-transition as a key step in progression and invasion of solid cancers ${ }^{12-14}$.

Immune cells have also been intensively studied and are now recognized as an essential component of the tumour microenvironment ${ }^{15}$. Indeed, certain tumours, most notably microsatellite-instable (MSI-high) CRC, are associated with a dense T-cell lymphocytic infiltrate, less tumour budding and better prognosis 16-18. Meanwhile, many works have led to the recognition of three lymphocyte markers: CD3+, CD8+ and CD45R0+ ${ }^{19-21}$ and the concept of Immunoscore 22, 6 . A validation study demonstrating Immunoscore to be a reliable predictor of recurrence risk has recently been published ${ }^{5}$.

To date, most investigations of tumour budding and T-cell infiltrates in CRC have been independent from each other. Introducing the concept of the 'attacker-defender model', a previous study examining a combined CD8+T-cell/tumour budding index demonstrated superior prognostic information than either marker alone ${ }^{23}$. Nevertheless, a systematic analysis of potential protagonists of the attacker-defender model has not yet been conducted. The aim of this study was to assess tumour budding in relationship to the T-cell infiltrate focusing on CD3+, CD8+ and CD45R0+ subpopulations (Budding/T-cell Score, BTS) in different tumour areas and to determine which elements lead to the best correlation with aggressive tumour biology and patient outcome. 


\section{Materials and Methods}

\section{Patient cohort}

A retrospective collective of 345 primary colorectal cancer patients treated by resection at the University Hospital of Bern between 2002 and 2014 was used for this study. Cases were rereviewed (A.L. and H.D.) based on the TNM $7^{\text {th }}$ edition (review performed prior to the publication of the $8^{\text {th }}$ edition). Assessed features (Table 1) include histological subtype, tumour location, $\mathrm{pT}$, $\mathrm{pN}, \mathrm{pM}$ and TNM stage (defined as $\mathrm{pT}, \mathrm{pN}$ and either $\mathrm{pM}$ or $\mathrm{cM}$ at the time of diagnosis), grade, lymphatic invasion, venous invasion, perineural invasion, Klintrup-Mäkinen score ${ }^{24}$, tumour budding scored by the ITBCC recommendations ${ }^{10}$ and by immunohistochemical pankeratin stains ('overall tumour budding, OTB') 25. Importantly, tumour budding had previously been assessed by both of these methods on whole tissue slides on these cases and was significantly associated with poorer survival 25, 26. Status of mismatch repair (MMR) proteins was assessed by immunohistochemistry of MLH1, MSH2, PMS2 and MSH6 as previously described 27 and classified as mismatch-repair deficient (nuclear loss of at least one marker) and proficient. Information on therapy and overall survival were obtained for all patients (mean and median follow-up time, 47.8 and 40.7 months, respectively). Information on synchronous or metachronous distant metastases was available for 324 patients $(\mathrm{cM} 0 / \mathrm{cM} 1$ in Table 1). No patients received preoperative therapy. The use of patient material was approved by the ethics commission of the canton of Bern (KEK 2017-01803, October 24, 2017).

\section{Next Generation Tissue Microarray construction}

This study was performed on a tissue microarray using the next generation tissue microarray (ngTMA $\left.{ }^{\circledR}\right)$ approach ${ }^{28}$. Annotations made from areas containing tumour tissue were selected in the highest budding areas. For each patient, three punches from different histological regions were investigated: tumour centre, tumour front, and tumour microenvironment (areas at the tumor/host interface containing tumor buds as well as non-tumoural cells) defined as previously described ${ }^{29}$ ( $\mathrm{n}=9$ spots per patient, core diameter $0.6 \mathrm{~mm}$ for all tumour areas).

\section{Double immunohistochemistry for CD8/pankeratin, CD3/ pankeratin and CD45R0/ pankeratin}


Three double immunostains were performed, namely the pankeratin cocktail AE1/AE3 to highlight tumour cells and CD8, CD3 or CD45RO, respectively. Double immunostains ngTMA blocks containing cores from tumour centre, front and microenvironment using the BOND-RX Automated System (Leica Microsystems GmbH, Newcastle, United Kingdom). ngTMA blocks were cut at $4 \mu \mathrm{m}$, deparaffinised and pre-treated with the Epitope Retrieval Solution 2 (Citratebuffer $\mathrm{pH} 8.8$ ) at $100 \mathrm{C}^{\circ}$ for $20 \mathrm{~min}$. After wash steps, peroxidase blocking was carried out for 4 min using the Bond Polymer Refine Detection Kit DC9800 (Leica Microsystems GmbH). Tissues were again washed, then incubated with primary antibody against AE1/AE3 with the dilution 1:200 (DakoCytomation, Glostrup, Denmark) for 30min. Subsequently, tissues were incubated with polymer for $15 \mathrm{~min}$ and then with DAB-Chromogen for 10min (Bond Polymer AP Red Detection Kit DS9305, Leica Microsystems GmbH). After washing, incubation was carried out with anti-CD8 (DakoCytomation, Glostrup, Denmark), anti-CD3 (Abcam, Cambridge) and antiCD45RO (Abcam, Cambridge) with the dilutions CD8 $1: 100$, CD3 $1: 400$, CD45RO $1: 5000$ for $30 \mathrm{~min}$ (reduced to $15 \mathrm{~min}$ in optimizing the CD45RO protocol) followed by application of AECsubstrate for $10 \mathrm{~min}$ and counterstaining with haematoxylin for $5 \mathrm{~min}$.

\section{Evaluation of tumour budding cells and T-cell infiltrates on digital slides}

Analysis of tumour buds and T-cell infiltrates on the double immunostains was performed on scanned slides (P250, 3DHistech, Budapest, Hungary) using the open source software QuPath ${ }^{30}$.

As algorithms for reliable automated digital assessment of tumour budding are still under development, buds were scored manually using an online tool 'Scorenado' especially developed for TMA scoring ${ }^{31}$, on dearrayed spots. Tumour budding was defined as an isolated tumour cell or a cluster of four or less tumour cells irrespective of location. Punches containing only stroma (no tumour) were excluded from further evaluation and therefore have no BTS scores.

For assessment of T-cell infiltrates with QuPath, automatically detected TMA spots underwent manual quality control and correction. Tissue areas were detected automatically and manual quality control was performed to remove artefacts. Stroma areas were defined by subtracting tumour areas from their parent tissue areas. Cell detection was performed within stroma areas and the detected cells subsequently classified as 'T-cells' and 'other' using a threshold classifier based on eosin staining. The procedure of digital assessment of T-cell infiltrates is depicted in Fig. 1. All 
scripts used for image analysis in QuPath are available under https://github.com/TranslationalResearchUnit/CRC-BTS.

\section{Calculation of Budding/T-cell scores (BTS)}

To calculate the density of T-cells, the digital count was divided by the corresponding stroma area. Tumour bud cell density was calculated by dividing the number of buds counted on Scorenado divided by the corresponding stroma area. The Budding/T-cell score (BTS) was defined as the number of tumour buds divided by the number of lymphocytes. The BTS was first calculated for each tissue spot individually. The BTS on a patient level was calculated for each combination of tumour area type and T-cell type as the mean of all BTSs on tissue spot level of the corresponding combination. As division by zero is not possible, the count of all tumour buds (numerator) and immune cells (denominator) was increased by +1 .

\section{Statistical analysis}

The association of the BTS scores with clinico-pathological features was obtained using the Wilcoxon rank sum test for binary features, the Kruskal-Wallis test for ordinal features, and the Spearman test for continuous features. All p-values (Supplementary file S2) were two-sided and considered significant when $\mathrm{p}<0.05$. These analyses were carried out using the SciPy library (version 1.1.0) in Python 3.5.1. For survival analysis a univariate Cox regression and a multivariate Cox regression (including $\mathrm{T}$-stage, $\mathrm{N}$-stage, $\mathrm{cM}$ stage and postoperative therapy) was performed, using the lifelines library (0.13.0) for Python 3.5.1. Hazard ratios (HR) and 95\%CI were obtained to determine the effect of each feature.

\section{Results}

\section{T-cell/budding count alone versus BTS and clinico-pathological features}

The distribution of all scores in different tumour areas is shown in Supplementary File S1. Associations between T-cell infiltrates, tumour budding scores and a combined BTS score are depicted in Fig. 3, with exact p-values in Supplementary File S2. Comparing the T-cell infiltrates, the number of significant statistical associations with clinico-pathological features for CD3 and CD8 were markedly higher than CD45RO both as single parameters and in a combined BTS score. Focusing on CD3 and CD8, infiltrates assessed in the tumour centre stood out with high 
associations with lower pT-stage $(\mathrm{p}<0.001$, both), lower $\mathrm{pN}$-stage $(\mathrm{p}=0.0085$ and $\mathrm{p}<0.001$, respectively), cM-stage ( $\mathrm{p}<0.001$ and $\mathrm{p}=0.0088)$, higher $\mathrm{pTNM}$ stage $(\mathrm{p}<0.001$, both), absence of lymphatic invasion $(p=0.0024$ and $p=0.0003)$ and blood vessel invasion $(p=0.0106$ and $p=0.009)$, higher KM-Score $(\mathrm{p}<0.001$, both), and lower tumour budding counts as assessed by the ITBCC method $(p=0.0005$ and $p=0.0042)$.

Tumour budding showed consistent significant associations in virtually all areas (centre, front, tumour microenvironment) with aggressive tumour biology, with higher $p T$-stage $(\mathrm{p}=0.0125$, $\mathrm{p}=0.0418$ and $\mathrm{p}=0.0062), \mathrm{pN}$-stage $(\mathrm{p}=0.0002, \mathrm{p}=0.0005$ and $\mathrm{p}<0.001)$, TNM-stage $(\mathrm{p}=0.0011$, $\mathrm{p}=0.0025$ and $\mathrm{p}<0.001$ ), lymphatic invasion, blood vessel invasion and perineural invasion. Importantly, correlations between tumour budding assessed on the TMA punches and by ITBCC and OTB methods were both highly significant ( $\mathrm{p}<0.001$, all areas)

For CD3 and CD8 BTS from the tumour centre, higher scores were associated with higher pTstage $(p=p<0.001$, both), higher $\mathrm{pN}$-stage $(\mathrm{p}=0.004$ and $\mathrm{p}<0.001$, cM-stage $(\mathrm{p}=0.0007$ and $\mathrm{p}=0.0015)$, pTNM-stage $(\mathrm{p}<0.001$, both), lymphatic invasion $(\mathrm{p}<0.001$, both), venous invasion $(p=0.001, p=0.006)$, perineural invasion $(p=0.0032$ and $p=0.0003)$, lower Klintrup-Mäkinen score $(p=0.0042$ and $p=0.0002)$, OTB $(p=0.0162$ and $p=0.0162)$ and ITBCC scores $(p<0.001$, both).

\section{BTS vs. immune cell/budding count alone as a predictor of lymph node metastasis and}

\section{survival}

In the context of clinically relevant endpoints (lymph node metastasis and overall survival), associations with T-cell infiltrates, tumour buds and BTS scores were assessed.

On univariate analysis, higher CD8 infiltrates in the tumour centre were associated with better OS $(\mathrm{p}=0.0002)$, followed by CD3 in the tumour centre $(\mathrm{p}=0.0016)$. The BTS showed centre and TME CD3 BTS as well as centre CD8 BTS to be associated with poorer survival ( $p=0.0437,0.0266$, and 0,0214 respectively). Budding on the TMA spots as a sole parameter was not significant for OS in this cohort. However, in multivariate analysis including $\mathrm{T}$-stage, $\mathrm{N}$-stage, cM stage and postoperative therapy, only higher centre CD3 BTS scores were significantly associated with poorer OS ( $p=0.012$, HR 1.218, 95\%CI: 1.044-1.419).

For predicting nodal metastases, only centre CD8 BTS scores were significant for predicting nodal metastases ( $\mathrm{p}<0.0001$, OR 1.466, 95\%CI: 1.115-1.928). 


\section{Discussion}

This study is the first analysis to explicitly examine T-cell populations and tumour budding cells in colorectal cancer as a combined Budding/T-cell Score in different tumour areas. Here, although T-cell infiltrates and tumour budding were both strongly associated with clinico-pathological features, a combined score was superior to either marker alone, especially for predicting nodal metastases and survival.

T-cell infiltrates in solid cancers have been the focus of many studies, and the recently validated Immunoscore has been demonstrated to provide valuable information on recurrence risk in nonmetastatic colon cancer ${ }^{5}$. However, Immunoscore may not completely reflect the biology of the tumour itself. Here, we study how the 'attacker-defender model' might enhance the estimation of tumour biology compared to tumour budding or T-cell infiltrates as separate parameters. For instance, it would be conceivable that additional quantification of T-cell infiltrates could counteract the effect of tumour budding. This may prove useful in clinical scenarios where tumour budding can be implemented to guide patient management, such as estimating the risk of nodal metastases in endoscopically resected pT1 CRC ${ }^{9,32}$ and in stage II CRC 33,3410 .

Our group has previously demonstrated superior prediction of survival when using a combined index of tumour buds and T-cells. For instance, a CD8/tumour budding index was shown to increase the prognostic effect of tumour budding ${ }^{23}$. Similarly, a combined assessment of CD8, FOXP3 and CD68 positive immune cells and tumour buds was also described to improve stratification of patients into prognostic risk groups ${ }^{35}$. Even histomorphological assessment of tumour-infiltrating lymphocytes and tumour buds may increase the impact of tumour budding alone, as recently suggested ${ }^{36}$.

Taking into account insights on the T-cell landscape of CRC, we focus on several well-established T-cell protagonists (CD3, CD8 and CD45RO) in different areas of the tumour. Interestingly, the most robust parameters in our assessment are CD3 and CD8 BTS scores originating from the tumour centre. Although traditionally assessed at the tumour front ${ }^{10}$, tumour budding within the tumour centre (intratumoural budding, ITB) is well-documented in the literature and strongly 
correlated with budding seen at the tumour front $25,37,38$. The fact that budding can be seen in the centre of a tumour strongly supports that dissemination does not occur only in regions rigidly defined as 'tumour front' and 'tumour microenvironment' 39 . Such areas of infiltrative tumour growth may also be highly convoluted and therefore also captured in regions corresponding to our 'tumour centre' punches. Finally, technical aspects such as levelling through TMA cores may have additionally contributed to a higher consistency of scores derived from the tumour centre. Nonetheless, practical implications of this finding include potential application of the BTS score in pre-operative rectal biopsies, where additional information on tumour spread may influence the decision to administer neoadjuvant therapy 40,41 .

From the T-cell counts, CD3 and CD8 counts were superior compared to CD45RO, which was also reflected in the BTS scores. Though regulatory T-cells have been implicated as an important player in the immune cell infiltrate in CRC 6,42,43, inconsistencies and background positivity in immunohistochemical stains have previously been reported 6,44 , leading to the exclusion of CD45RO from Immunoscore ${ }^{6}$. The diminished performance of CD45RO-based scores here is most likely also due to this phenomenon despite efforts to improve the quality of the immunostain, indicating that this marker may not be suitable for quantification performed by visual scoring or digital assessment.

Our proposal of the BTS aims to reflect the 'attacker' and 'defender' as aggressive and protective forces in CRC. However, further studies are required to validate whether these opposite markers can indeed be united in a combined score. Although our study was performed on a multi-punch TMA including several punches from different tumour areas, due to the nature of this approach evaluated tissue originated from pre-selected areas. This would account for the lack of association between tumour buds scored on the TMA spots and survival, as tumour budding assessed on whole tissue slides from this collective was associated with poorer overall survival (by both OTB and ITBCC methods $\left.{ }^{25}, 26\right)$. Validation studies may be performed on whole tissue slides to optimize the selection of regions most suitable for BTS assessment.

One of the novel aspects of this study is the assessment of T-cell infiltrates using open-source software. The implementation of such methods will likely be able to replace cumbersome manual assessment in the near future. As digital scoring of tumour buds can also be anticipated, a fully 
automated BTS-algorithm could be developed. The BTS is not meant to replace tumour budding and Immunoscore as robust biomarkers in $\mathrm{CRC}$, but to further underline and reflect their importance alongside other pathological parameters. Potential applications of the BTS include clinical scenarios where a precise estimation of tumour biology is required to guide patient management, such as in endoscopically resected pT1 tumours, stage II CRC and pre-operative rectal biopsies. Our results warrant further study and development of the BTS in CRC.

\section{Author Contributions and Acknowledgements}

HD and LC performed manual scoring, ME and SR conducted digital analyses. AL and HD designed the study. ME and IZ performed statistical analysis. HD, ME and LC drafted the manuscript. AL, AB, SR and IZ critically reviewed the manuscript.

Additionally, the authors thank the members of the Translational Research Unit, Institute of Pathology, University of Bern for their excellent technical support, especially Dr. José Galvan for his assistance in optimizing the double immunostains used for this project. No external funding was required for this project.

\section{References}

1. Jessup JM GR, Asare EA, Benson AB, Brierly JD, Chang GJ et al. . Colon and rectum. In MB A ed. Ajcc cancer staging manual, 8th edition: Springer Nature, 2017 251-274.

2. Brierly JD GM, Wittekind C. . Tnm classification of malignant tumors. 8th ed. Oxford, UK: Wiley Blackwell, 2017.

3. Mitrovic B, Schaeffer DF, Riddell RH, Kirsch R. Tumor budding in colorectal carcinoma: Time to take notice. Mod Pathol 2012;25;1315-1325.

4. De Smedt L, Palmans S, Sagaert X. Tumour budding in colorectal cancer: What do we know and what can we do? Virchows Arch 2016;468;397-408.

5. Pages F, Mlecnik B, Marliot F et al. International validation of the consensus immunoscore for the classification of colon cancer: A prognostic and accuracy study. Lancet 2018;391;2128-2139.

6. Galon J, Mlecnik B, Bindea G et al. Towards the introduction of the 'immunoscore' in the classification of malignant tumours. J Pathol 2014;232;199-209.

This article is protected by copyright. All rights reserved 
7. van Wyk HC, Park JH, Edwards J, Horgan PG, McMillan DC, Going JJ. The relationship between tumour budding, the tumour microenvironment and survival in patients with primary operable colorectal cancer. Br J Cancer 2016;115;156-163.

8. Rogers AC, Winter DC, Heeney A et al. Systematic review and meta-analysis of the impact of tumour budding in colorectal cancer. Br J Cancer 2016;115;831-840.

9. Bosch SL, Teerenstra S, de Wilt JH, Cunningham C, Nagtegaal ID. Predicting lymph node metastasis in pt1 colorectal cancer: A systematic review of risk factors providing rationale for therapy decisions. Endoscopy 2013;45;827-834.

10. Lugli A, Kirsch R, Ajioka Y et al. Recommendations for reporting tumor budding in colorectal cancer based on the international tumor budding consensus conference (itbcc) 2016. Mod Pathol 2017;30;1299-1311.

11. Kakar S SC, Berho ME et al. College of american pathologists: Protocol for the examination of specimens from patients with primary carcinoma of the colon and rectum. Edition 4.0.0.1. . http://www.cap.org/ShowProperty?nodePath=/UCMCon/Contribution\%20Folders/WebContent/pdf/cpcolon-17protocol-4001.pdf. Access Date: May 22, 2017.

12. De Smedt L, Palmans S, Andel D et al. Expression profiling of budding cells in colorectal cancer reveals an emt-like phenotype and molecular subtype switching. Br J Cancer 2017;116;58-65.

13. Dawson $\mathrm{H}$, Lugli A. Molecular and pathogenetic aspects of tumor budding in colorectal cancer. Front Med (Lausanne) 2015;2;11.

14. Zlobec I, Lugli A. Epithelial mesenchymal transition and tumor budding in aggressive colorectal cancer: Tumor budding as oncotarget. Oncotarget 2010;1;651-661.

15. Hanahan D, Coussens LM. Accessories to the crime: Functions of cells recruited to the tumor microenvironment. Cancer Cell 2012;21;309-322.

16. De Smedt L, Lemahieu J, Palmans S et al. Microsatellite instable vs stable colon carcinomas: Analysis of tumour heterogeneity, inflammation and angiogenesis. Br J Cancer 2015;113;500-509.

17. Nosho K, Baba Y, Tanaka N et al. Tumour-infiltrating t-cell subsets, molecular changes in colorectal cancer, and prognosis: Cohort study and literature review. J Pathol 2010;222;350-366.

18. Jass JR, Barker M, Fraser L et al. Apc mutation and tumour budding in colorectal cancer. J Clin Pathol 2003;56;69-73.

19. Mlecnik B, Tosolini M, Kirilovsky A et al. Histopathologic-based prognostic factors of colorectal cancers are associated with the state of the local immune reaction. J Clin Oncol 2011;29;610-618.

20. Broussard EK, Disis ML. Tnm staging in colorectal cancer: T is for $t$ cell and $\mathrm{m}$ is for memory. $J$ Clin Oncol 2011;29;601-603.

This article is protected by copyright. All rights reserved 
21. Fridman WH, Pages F, Sautes-Fridman C, Galon J. The immune contexture in human tumours: Impact on clinical outcome. Nat Rev Cancer 2012;12;298-306.

22. Galon J, Pages F, Marincola FM et al. The immune score as a new possible approach for the classification of cancer. J Trans/ Med 2012;10;1.

23. Lugli A, Karamitopoulou E, Panayiotides I et al. Cd8+ lymphocytes/tumour-budding index: An independent prognostic factor representing a 'pro-/anti-tumour' approach to tumour host interaction in colorectal cancer. Br J Cancer 2009;101;1382-1392.

24. Klintrup K, Makinen JM, Kauppila S et al. Inflammation and prognosis in colorectal cancer. Eur J Cancer 2005;41;2645-2654.

25. Rieger G, Koelzer VH, Dawson HE et al. Comprehensive assessment of tumour budding on cytokeratin stains in colorectal cancer. Histopathology 2017.

26. Dawson H GF, Träger P, Berger, MD, Studer P, Bruegger P, Zlobec I, Inderbitzin D, Lugli A. Validation of the international tumor budding consensus conference (itbcc 2016) recommendations on tumor budding in stage i-iv colorectal cancer. Hum Pathol 2018 (in press).

27. Dawson $\mathrm{H}$, Koelzer $\mathrm{VH}$, Lukesch $\mathrm{AC}$ et al. Loss of $\mathrm{cdx} 2$ expression in primary tumors and lymph node metastases is specific for mismatch repair-deficiency in colorectal cancer. Front Oncol 2013;3;265.

28. Zlobec I, Koelzer VH, Dawson H, Perren A, Lugli A. Next-generation tissue microarray (ngtma) increases the quality of biomarker studies: An example using $\mathrm{cd} 3, \mathrm{~cd} 8$, and $\mathrm{cd} 45 \mathrm{ro}$ in the tumor microenvironment of six different solid tumor types. J Transl Med 2013;11;104.

29. Koelzer VH, Karamitopoulou E, Dawson H, Kondi-Pafiti A, Zlobec I, Lugli A. Geographic analysis of rkip expression and its clinical relevance in colorectal cancer. Br J Cancer 2013;108;2088-2096.

30. Bankhead P, Loughrey MB, Fernandez JA et al. Qupath: Open source software for digital pathology image analysis. Sci Rep 2017;7;16878.

31. Eichmann M, Reinhard, S., Zlobec I. . Scorenado: An efficient and user-friendly visual assessment tool. 4th European Congress on Digital Pathology (ECDP 2018). Helsinki, 2018.

32. Beaton C, Twine CP, Williams GL, Radcliffe AG. Systematic review and meta-analysis of histopathological factors influencing the risk of lymph node metastasis in early colorectal cancer. Colorectal Dis 2013;15;788-797.

33. Petrelli F, Pezzica E, Cabiddu M et al. Tumour budding and survival in stage ii colorectal cancer: A systematic review and pooled analysis. J Gastrointest Cancer 2015;46;212-218.

34. Benson A, Venook, AP, Cederquist L et al. National comprehensive cancer network. Clinical practice guidelines in oncology (nccn guidelines): Colon cancer.

This article is protected by copyright. All rights reserved 
Https://www.Nccn.Org/professionals/physician_gls/default.Aspx. Access date: March 18, 2018. https://www.nccn.org/professionals/physician_gls/default.aspx. Access Date: March 18, 2018.

35. Zlobec I, Minoo P, Terracciano L, Baker K, Lugli A. Characterization of the immunological microenvironment of tumour buds and its impact on prognosis in mismatch repair-proficient and deficient colorectal cancers. Histopathology 2011;59;482-495.

36. Lang-Schwarz C, Melcher B, Haumaier F et al. Budding and tumor infiltrating lymphocytes combination of both parameters predicts survival in colorectal cancer and leads to new prognostic subgroups. Hum Pathol 2018.

37. Zlobec I, Hadrich M, Dawson $\mathrm{H}$ et al. Intratumoural budding (itb) in preoperative biopsies predicts the presence of lymph node and distant metastases in colon and rectal cancer patients. Br J Cancer 2014;110;1008-1013.

38. Lugli A, Vlajnic T, Giger O et al. Intratumoral budding as a potential parameter of tumor progression in mismatch repair-proficient and mismatch repair-deficient colorectal cancer patients. Hum Pathol 2011;42;1833-1840.

39. Bronsert $\mathrm{P}$, Enderle-Ammour K, Bader $\mathrm{M}$ et al. Cancer cell invasion and emt marker expression: A three-dimensional study of the human cancer-host interface. J Pathol 2014;234;410-422.

40. Zlobec I, Borner M, Lugli A, Inderbitzin D. Role of intra- and peritumoral budding in the interdisciplinary management of rectal cancer patients. Int J Surg Oncol 2012;2012;795945.

41. Rogers AC, Gibbons D, Hanly AM et al. Prognostic significance of tumor budding in rectal cancer biopsies before neoadjuvant therapy. Mod Pathol 2014;27;156-162.

42. Hu G, Wang S. Tumor-infiltrating cd45ro(+) memory t lymphocytes predict favorable clinical outcome in solid tumors. Sci Rep 2017;7;10376.

43. Pages F, Berger A, Camus $\mathrm{M}$ et al. Effector memory t cells, early metastasis, and survival in colorectal cancer. N Engl J Med 2005;353;2654-2666.

44. Koelzer VH, Lugli A, Dawson $\mathrm{H}$ et al. Cd8/cd45ro t-cell infiltration in endoscopic biopsies of colorectal cancer predicts nodal metastasis and survival. J Transl Med 2014;12;81.

This article is protected by copyright. All rights reserved 
Feature

Gender $(n=345)$

Patient age $(\mathrm{n}=345)$

Histological subtype $(n=326)$

Tumour location $(n=320)$

$\mathrm{pT}(\mathrm{n}=342)$

Male
Female
Mean, median
Adenocarcinom
Mucinous
Other
Left-sided
Right-sided
Rectum
pT1-2

Freq N (\%) or

Mean, median

209 (60.6)

$136(39.4)$

$69.5,70.9$

$283(86.8)$

$37(11.3)$

$6(1.8)$

$142(44.4)$

118 (36.9)

$60(18.8)$

59 (17.3)
Tables

Table 1: Patient characteristics $(n=345)$ 


\begin{tabular}{|c|c|c|}
\hline & pT3-4 & $283(82.2)$ \\
\hline \multirow[t]{2}{*}{$\mathrm{pN}(\mathrm{n}=342)$} & pNO & $160(46.7)$ \\
\hline & $\mathrm{pN} 1-2$ & $182(53.3)$ \\
\hline $\begin{array}{l}\text { Number of examined lymph nodes } \\
(\mathrm{n}=338)\end{array}$ & Mean, median & $24.2,21.0$ \\
\hline \multirow[t]{2}{*}{ Distant metastases $(\mathrm{n}=324)$} & $\mathrm{cM} 0$ & $238(73.5)$ \\
\hline & cM1 & $86(26.5)$ \\
\hline \multirow[t]{4}{*}{ p/cTNM-Stage $(n=339)$} & Stage I & $37(10.9)$ \\
\hline & Stage II & $126(37.2)$ \\
\hline & Stage III & $120(35.4)$ \\
\hline & Stage IV & $56(16.5)$ \\
\hline \multirow[t]{2}{*}{ Tumour grade $(\mathrm{n}=332)$} & G1-2 & $259(78.0)$ \\
\hline & G3 & $73(22.0)$ \\
\hline $\begin{array}{l}\% \quad \text { expanding tumour border } \\
(\mathrm{n}=302)\end{array}$ & Mean, median & $45.9,50.0$ \\
\hline Number of buds ITBCC $(\mathrm{n}=106)$ & Mean, median & $8.1,6.0$ \\
\hline OTB mean $(\mathrm{n}=186)$ & Mean, median & $11.2,8.0$ \\
\hline \multirow[t]{2}{*}{ Lymphatic invasion $(\mathrm{n}=310)$} & L0 & $98(31.6)$ \\
\hline & L1 & $212(68.4)$ \\
\hline \multirow[t]{2}{*}{ Venous invasion $(\mathrm{n}=293)$} & V0 & $141(48.1)$ \\
\hline & V1-2 & $152(51.9)$ \\
\hline \multirow[t]{2}{*}{ Perineural invasion $(\mathrm{n}=300)$} & Pn0 & $247(80.7)$ \\
\hline & Pn1 & $59(19.3)$ \\
\hline \multirow[t]{4}{*}{ Klintrup-Mäkinen ( $\mathrm{n}=305)$} & 0 & $25(8.2)$ \\
\hline & 1 & $129(42.3)$ \\
\hline & 2 & $119(39.0)$ \\
\hline & 3 & $32(10.5)$ \\
\hline \multirow[t]{2}{*}{ Postoperative therapy $(\mathrm{n}=284)$} & No & $196(69.0)$ \\
\hline & Yes & $88(31.0)$ \\
\hline MMR status ( $\mathrm{n}=190)$ & Proficient & $164(86.3)$ \\
\hline & Deficient & $26(13.7)$ \\
\hline
\end{tabular}


Abbreviations: ITBCC: International Tumor Budding Consensus Conference; OTB : Overall tumor budding; MMR : Mismatch repair

\section{Figure Legends}

Fig. 1: Low (A-C) and high (D-F) BTS scores for each double immunostain. A+D: CD3/Pankeratin, B+E: CD8/Pankeratin, C+F: CD45RO/Pankeratin (images all taken between 1520x). Selected tumour buds marked by asterisks, selected T-cells marked by arrows (A-F). The $\mathrm{CD} 45 \mathrm{RO} /$ Pankeratin immunostain shows extensive non-specific staining which was still present after protocol optimization.

Fig. 2: Digital analysis of TMA spots $(0.6 \mathrm{~mm}$ in diameter) and assessment of T-cell infiltrates. a) Double immunostain (CD8(red)/Pankeratin(brown)) before and b) after tissue separation. 'Stroma' was defined as the non-epithelial, non-T-cell area (nuclei annotated in green).

Fig. 3: Plot of associations between T-cell infiltrates, tumour buds and Budding/T-cell scores with clinico-pathological features and overall survival. Increased numbers of T-cells are associated with 
more favourable tumour biology, whereas increased numbers of tumour buds and higher Budding/T-cell scores are associated with aggressive tumour biology. Abbreviations: TME: tumour microenviroment, BTS: Budding/T-cell scores, KM-Score: Klintrup-Mäkinen score, OTB: overall tumour budding, ITBCC: International tumour budding consensus conference, OS: overall survival

\section{Supplementary Files:}

S1: Distribution of T-cell and tumour bud counts and BTS scores in different tumour areas

S2: Depiction of Fig. 3 with exact p-values 
a)

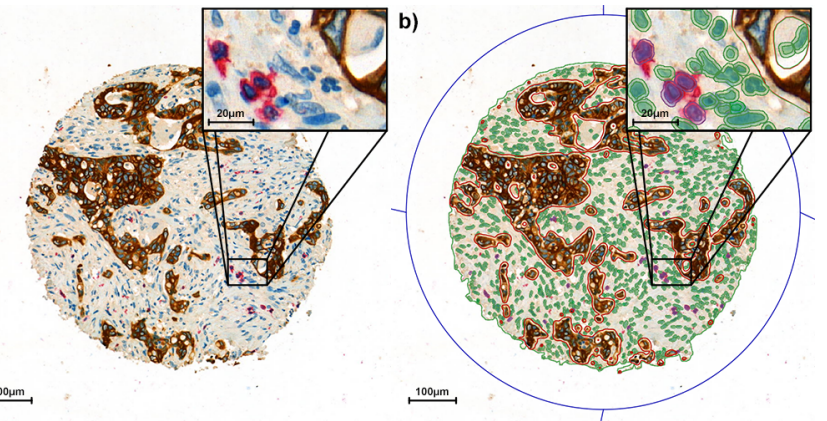

his_14006_f2.tif

This article is protected by copyright. All rights reserved 


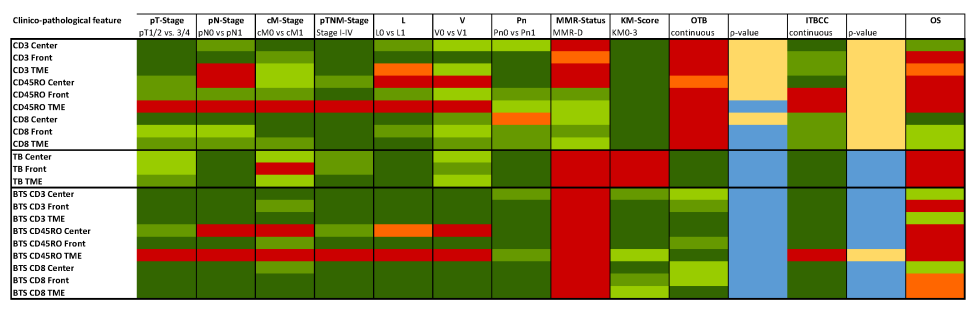

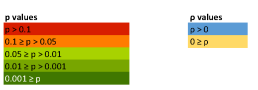

his_14006_f3.tif 